\title{
Progressive plastic changes in the hand representation of the primary motor cortex parallels incomplete recovery from a unilateral section of the corticospinal tract at cervical level in monkeys
}

\author{
Eric Schmidlin ${ }^{1, *}$, Thierry Wannier ${ }^{1,2, *}$, Jocelyne Bloch $^{3}$ and Eric M. Rouiller ${ }^{1}$ \\ * the first two authors contributed equally to this study.
}

(1) Unit of Physiology and Program in Neurosciences, Department of Medicine, Faculty of sciences, University of Fribourg, Chemin du Musée 5, CH-1700 Fribourg, Switzerland.
(2) Brain Research Institute, Dept. Neuromorphology, University and ETH Zurich, Winterthurerstr. 190, CH-8057 Zürich, Switzerland.

(3) Dept. of Neurosurgery, Neurosurgery Clinic, University Hospital of Lausanne, Rue du Bugnon, $\mathrm{CH}-1011$ Lausanne, Switzerland.

Running title: changes of M1 hand representation after cervical lesion

Text pages: 26

Words (without abstract, references and Fig. legends): 6752

Abstract: 242 words

Tables: 0

Figures: 4

\section{Acknowledgements}

The authors wish to thank the technical assistance of Véronique Moret, Françoise Tinguely and Christine Roulin (histology and behavioral evaluations), Josef Corpataux and Bernard Morandi (animal house keeping), André Gaillard (mechanics), Bernard Aebischer (electronics), Laurent Monney (informatics).

Grant Sponsors: Swiss National Science Foundation, grants No 31-43422.95, 4038-43918, 31-61857.00 (EMR); Novartis Foundation; The National Centre of Competence in Research (NCCR) on "Neural plasticity and repair".

* Address for correspondence: Prof. Eric M. Rouiller, Unit of Physiology, University of Fribourg, Chemin du Musée 5, CH-1700 Fribourg, Switzerland. Phone: 00412630086 09. Fax: 0041 2630096 75. E-mail: Eric.Rouiller@unifr.ch 


\section{Abstract}

After a sub-total hemi-section of the cervical cord at level C7/C8 in monkeys, a paralysis of the homolateral hand is rapidly followed by an incomplete recovery of manual dexterity, reaching a plateau after about 40-50 days, whose extent appears related to the size of the lesion. During a few days after the lesion, the hand representation in the contralateral motor cortex disappeared, replaced by representations of either face or more proximal body parts. Later, however, following a time course (about 40 days) consistent with the functional recovery, progressive plastic changes in the contralateral motor cortex took place, as demonstrated by a progressive re-appearance of digit movements elicited by intracortical microstimulation. These progressive plastic changes, which parallel the functional recovery, correspond to a re-installation of a hand representation, though substantially diminished in size as compared to pre-lesion. Regarding the functional recovery, the motor cortex (including the re-established hand area) contralateral to the unilateral cervical cord lesion played a crucial role in re-establishing control on finger movements, as assessed by reversible inactivation experiments. In contrast, the motor cortex ipsilateral to the cervical cord lesion, with largely intact projections to the spinal cord, did not contribute significantly to the recovered movements by the affected hand. These observations indicate that the CS fibers spared by the lesion are not sufficient, at least in their pre-lesion condition, to control the motoneurones innervating the digit muscles and that the pathways conveying signals from the contralateral M1 underwent reorganization.

\section{Theme G: Motor system and sensorimotor integration}

\section{Topic: Cortex}

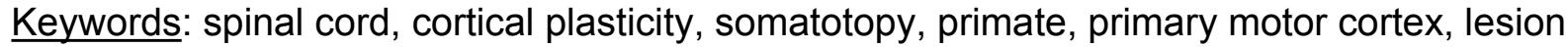




\section{Introduction}

Numerous studies in subhuman primates have investigated the effects of lesioning the corticospinal (CS) system either at its origin (cortical lesion; see [30] for review), along its course at the level of the pyramids (unilateral or bilateral; see [11,12,22 and 13 for review]) or closer to the termination zone in the spinal cord itself. The deficits associated with the latter segmental lesion were investigated in a few studies in monkeys $[1,4,7,8,15]$. Two elegant reports by Galea and Darian-Smith $[7,8]$ described the deficits and recovery of motor control associated with a hemisection of the cervical spinal cord at C3 level in new born and juvenile monkeys. Following a severe flaccid hemi-paresis of the forelimb on the side of the section, there was a substantial recovery of manual dexterity (grasping of an object using the precision grip) after about $60-90$ days. However, some deficits persisted: modified reaching trajectories, loss of pre-shaping prior grasping and weakening of oppositional forces between thumb and index finger [8].

The work of Galea and Darian-Smith $[7,8]$ did not address functional changes induced in the hand-representation of the primary motor cortex (M1) contralateral to the spinal lesion (termed the "contralateral M1" in the following). In the present study, the direct accessibility of the contralateral M1 to the spinal circuits controlling hand and finger movements was assessed using intracortical microstimulation (ICMS) mapping of M1 and compared to the time course of behavioral recovery. So far, most studies addressing the issue of cortical map plasticity in relation to a lesion (cortical or at the periphery) established the cortical map at two time points. The pre-lesion cortical map was compared to a post-lesion map, usually established after the functional recovery had reached a stable level $[31,32,39]$. In one study of the reorganization of the somatosensory cortex after spinal cord injury, cortical maps were established at several time points but in the absence of functional recovery curves $[16,17]$. A novel contribution of the present study is to provide, on a weekly time interval, a correlation between the functional recovery curve and progressive plastic changes of the hand-representation of the contralateral M1. In other words, our goal was to investigate to what extent progressive plastic changes of cortical map parallel recovery of manual dexterity during the weeks following the lesion. 


\section{Materials and Methods}

Detailed descriptions of surgical procedures (anesthesia, physiological monitoring of the animal, implantation of chronic chamber above M1, post-operative care) are available in previous reports from this laboratory $[19,20,24,25]$. The experiments were conducted in two young adult male (3-4 years old) Rhesus monkeys (Macaca mulatta), Mk1 and Mk2, weighing around 4 kg. Surgical procedures and animal care were conducted in accordance with the Guide for the Care and Use of Laboratory Animals (ISBN 0-309-05377-3; 1996) and approved by local veterinary authorities. The manual dexterity of each hand was assessed in the two monkeys using our modified "Brinkman board" task, as described in detail earlier [25,36]. Briefly, tests were done using a Perspex board $(10 \mathrm{~cm} \times 20 \mathrm{~cm})$ containing 50 randomly distributed holes; 25 holes were oriented horizontally and 25 vertically. The dimensions of the holes $(15 \mathrm{~mm}$ long, $8 \mathrm{~mm}$ wide and 6 $\mathrm{mm}$ deep) allowed the penetration of the index finger and the thumb to retrieve a food pellet by performing the so-called "precision grip". The monkeys performed this manual prehension dexterity task alternatively with one or the other hand, 4 to 5 times per week for several months before and several months after the spinal cord lesion. One to two weekly sessions were recorded on a video tape. An attempt was considered as successful when the monkey grasped a pellet and transported it to the mouth. The behavioral score was defined as the number of slots successfully retrieved within $1 \mathrm{~min}$. A daily behavioral session typically lasted for 15 to 20 minutes.

The somatotopic organization in and around the hand area in M1 was established based on daily ICMS sessions, as reported earlier $[25,35,36]$. Standard parameters of stimulation were used: $35 \mathrm{~ms}$ duration trains of 12 electric monophasic pulses $(0.2 \mathrm{~ms})$ presented once every 2 seconds, through a tungsten microelectrode (FHC, Maine, USA) with an impedance of $0.1 \mathrm{MOhms}$ and a tip of about 20-30 $\mu \mathrm{m}$. During the ICMS session (about 45 minutes), the monkey was awake, not performing any task, but the hand/arm contralateral to the stimulated M1 was constantly manipulated by one of the two experimenters. The same experimenter also checked movements elicited in the face, the anterior part of the trunk and the ipsilateral hand/arm. The other experimenter adjusted the intensity of stimulation and checked for movements in the back or neck. 
ICMS mapping was performed on both hemispheres. Depending on how quiet the animal was, between 1 and 4 electrode penetrations were typically performed within one ICMS session.

To unilaterally lesion the cervical cord, the following surgical procedures were applied. Anaesthesia was induced by intramuscular injection of ketamine (Ketalar®; Parke-Davis, 5 mg/kg, i.m.). Atropine was injected i.m. $(0.05 \mathrm{mg} / \mathrm{kg})$ to reduce bronchial secretions. Before surgery, the animal was treated with the analgesic Carprofen (Rymadil $囚, 4 \mathrm{mg} / \mathrm{kg}$, s.c.). An intravenous catheter was placed in the femoral vein for continuous perfusion with a mixture of $1 \%$ propofol (Fresenius ${ }^{\circledR}$ ) and a $4 \%$ glucose solution (1 volume of Propofol and 2 volumes of glucose solution), inducing a deeper anaesthesia. The animal was then placed in a stereotaxic framework. During the surgery under aseptic conditions, the following parameters were monitored: heart rate, respiration rate, expired $\mathrm{CO}_{2}$, arterial $\mathrm{O}_{2}$ saturation and body temperature. Usually, the level of anaesthesia remained deep and stable at a rate of venous perfusion of the Propofol/glucose mixture of 0.1 $\mathrm{ml} / \mathrm{min} / \mathrm{kg}$. An extra intravenous bolus of $0.5 \mathrm{mg}$ of ketamine diluted in saline $(0.9 \%)$ was added at potentially more painful steps of the surgical procedure, such as laminectomy. Placed in a ventral decubitus position, the spinal processes from $\mathrm{C} 2$ to Th1 were exposed. The paravertebral muscles were retracted and the laminae of $\mathrm{C} 6, \mathrm{C} 7$ and Th1 were dissected. A complete C6 laminectomy and an upper $\mathrm{C} 7$ hemi-laminectomy were then performed. The ligamentum flavum was removed. The dura mater was exposed and incised longitudinally. Under the microscope, the dorsal root entry zones were easily identified. To complete the left unilateral section of the spinal cord at the $\mathrm{C} 7 / \mathrm{C} 8$ border, the dorsal root entry zone was the most medial landmark. From this target, a surgical blade (no 11, Paragon $®$ ) was inserted $4 \mathrm{~mm}$ in depth perpendicularly to the spinal cord, and the section was prolonged laterally to completely cut the dorsolateral funiculus. From previously available anatomical material, the rostro-caudal level where the dorsal rootlets entering respectively the $7^{\text {th }}$ and the $8^{\text {th }}$ cervical spinal segments meet was determined, corresponding to the rostral zone of the spinal portion covered by the $6^{\text {th }}$ cervical lamina. The aimed lesion was located caudal with respect to the pool of biceps motoneurones but rostral to the pool of triceps, forearm and hand muscle motoneurones [18]. The muscles and the skin were sutured. The animal usually recovered from anesthesia 15-30 minutes after interruption of the perfusion with propofol 
and was treated post-operatively with an antibiotic (Ampiciline 10\%, $30 \mathrm{mg} / \mathrm{kg}$, s.c.). Additional doses of Carprofen were given daily during one week (pills of Rymadil mixed with food). After the spinal lesion, the animal was kept alone in a separate cage for a couple of days, to allow better conditions for recovery than the usual group housing with other monkeys.

The behavioral evaluation was pursued along a period of 3-5 months post-lesion, exhibiting a clear saturating effect on the incomplete recovery process, reflected by the plateau of the score of manual dexterity. At that stage (plateau), sessions of reversible inactivation of M1 in either hemisphere using muscimol were conducted, as previously described in detail $[19,25]$. Briefly, three ICMS penetration sites were usually selected in the hand area of M1 in one or the other hemisphere (see Fig. $2 \mathrm{~A}$ ) and the GABA-agonist muscimol ( $1 \mu \mathrm{g}$ in $1 \mu \mathrm{l}$ saline) was infused at two depths along each penetration (distant from each other by 2-3 mm). In Mk1, two muscimol inactivation sessions were conducted on each hemisphere, 6 weeks apart. In each inactivation session, the total volume of muscimol injected was $18 \mu \mathrm{l}$. In Mk2, only one muscimol inactivation session was conducted on each hemisphere (three days apart): three penetrations were performed on the contralesional hemiphere (total volume $18 \mu \mathrm{l}$ ), whereas $12 \mu \mathrm{l}$ were infused along two penetrations in the ipsilesional hemisphere. In both monkeys, the muscimol infusion sites in the ipsilesional hemisphere were also selected based on ICMS data (not shown). The efficacy of such reversible inactivation protocol has been demonstrated previously, together with control experiments in which only saline was injected [19].

\section{Results}

\subsection{Cervical hemi-section}

The lesion site was reconstructed from camera lucida drawings of individual consecutive sections of the spinal cervical cord, cut at $50 \mu \mathrm{m}$ in the saggital plane, collected in 3 series, and stained for Nissl or the marker SMI-32 (see [24]). An alignment of the drawings allowed reconstruction of the location and extent of the lesion on a frontal view of the spinal cervical cord (Fig. 1A). In the first animal (Mk1; left panel in Fig. 1A), the knife penetrated near the insertion site of the dorsal rootlets and travelled ventrally down to the ventral side of the spinal cord. It cut most 
of the left dorsolateral funiculus, the lateral part of the ventral horn and the white matter lying immediately ventrally to the latter. The dorsal, the ventromedial and lateral part of the ventrolateral funiculi were preserved. This knife cut thus sectioned most, if not all, of the CS fibres descending through the left dorsolateral funiculus, but preserved the fibres on the opposite side. In the second animal (Mk2; right panel of Fig. 1A), the section was larger and only the dorsal and the ventromedial funiculi were preserved on the lesioned side. Rostro-caudally, the maximum width of the scar covered a distance of up to $1 \mathrm{~mm}$ (see [37]).

\subsection{Behavioral data}

A few hours after the surgery aimed at unilaterally lesioning the cervical cord, the monkeys sat in the cage, showing a prominent stretching of the hindlimb ipsilateral to the lesion. As a result the animal was strongly handicapped for maintaining the standing posture and for changing position in the cage. The ipsilateral forelimb was maintained in a flexed position at the elbow, the fingers were all flexed and unused when food was presented to the animal. Movements of the ipsilateral shoulder were preserved. In contrast, no deficit was observed for the forelimb and hindlimb contralateral to the lesion. A few hours later, if forced to use the affected hand, the animal was able to catch a large piece of fruit by pushing further and together the flexed fingers in direction of the palm of the hand. No independent movement of the fingers was possible for several days.

As shown for Mk2 (Fig. 1B), the behavioral score reflecting the manual dexterity of the ipsilateral hand dropped to zero during a period of 2-3 weeks post-lesion. Then, an incomplete recovery of manual dexterity took place during 3-4 more weeks, saturating at a level of about 20$30 \%$ of the pre-lesion score, although there was some variability from one session to the next. In contrast, the behavioral score for the contralateral hand was not dramatically affected by the lesion, although it tended to be more variable across sessions (Fig. 1B). A comparable time course of recovery was observed in MK1, although the recovered manual dexterity score reached $70 \%$ of the pre-lesion score. The recovery in Mk1, although still incomplete, was clearly higher than in Mk2, as expected for a smaller lesion (Fig. 1A). 


\subsection{Mapping of M1 hand area before and after cervical cord lesion: ICMS data}

Although the present report is focused on subtle and progressive changes of the motor map in M1 taking place during the few weeks following the lesion, it is imperative to first describe the detailed properties of the motor map as it appears before the lesion. The hand area of M1 was thus extensively mapped in daily ICMS sessions taking place during the two months preceding the lesion. In the present paragraph, the pre-lesion motor map will be compared to the motor map reestablished extensively 2 and 4.5 months after the lesion in Mk1 and Mk2, respectively. The issue of the progressive changes taking place shortly after the lesion during the recovery period will be addressed below in paragraph 3.4 .

\subsubsection{Before lesion}

On a surface map of M1 (Fig. 2A, left column), each electrode penetration is represented at its corresponding position by a symbol indicating the body territory activated at the lowest ICMS current still eliciting a movement. Diamonds indicate the locations where the most excitable stimulation point along the electrode track induced a movement of digits. In the left column, the solid line surrounding the diamonds thus delineates the hand representation in the contralateral M1. In Mk2, the position of the chronic chamber allowed to investigate more rostral territories than in Mk1. As a result, a second, smaller hand area located more rostrally was observed in Mk2, which corresponds most likely to a territory in the premotor cortex (PM) where ICMS elicited digit movements, but at higher threshold than in the M1 hand area. In both monkeys (Fig. 2A, left column), the M1 hand area was surrounded by territories where ICMS at the lowest current elicited more proximal movements of the forelimb (wrist, elbow, shoulder) or movements of face muscles.

\subsubsection{After lesion}

The post-lesion ICMS maps, established after the recovery had reached a plateau, are shown in the right column of Figure 2A. Most ICMS sites effective before the lesion remained micro-excitable after the lesion. However, there were dramatic changes with respect to the precise body territory activated at a given ICMS site. These changes affected mainly the hand areas in both M1 and PM, which occupy post-lesion a significantly diminished cortical surface. In Mk1, with 
a section presumably interrupting most of the CS tract but preserving considerable amount of the lateral and ventral spinal white matter (Fig. 1A), the M1 hand area surface decreased from about $72 \mathrm{~mm}^{2}$ (pre-lesion) to $24 \mathrm{~mm}^{2}$ (post-lesion), thus representing a drop of $67 \%$. In Mk2, the lateral and ventral funiculi were nearly completely sectioned, resulting in an even more dramatic decrease of the surface of $\mathrm{M} 1$ hand area, dropping from $36 \mathrm{~mm}^{2}$ to $4 \mathrm{~mm}^{2}$ ( $89 \%$ decrease). In this animal, the rostral hand area in PM was even more affected since no movements at all could be elicited after the lesion. The M1 hand areas remaining after the lesion are delineated by a dashed contour in the right column of Fig. 2A. Territories separating the dashed contour from the solid contour are sites where ICMS elicited digits movements before the lesion, replaced by movements of other muscles (grey symbols) or non micro-excitable sites (X) post-lesion. Most of the sites addressing other muscles post-lesion elicited movements of face or wrist muscles. In other words, some of the pre-lesion hand points have been filled by adjacent body territories. There were also changes of territories outside the pre-lesion hand areas, but clearly less dramatic.

In the above computation leading to $67 \%$ and $89 \%$ decrease of hand area in the ICMS surface map observed as a result of the lesion in Mk1 and Mk2, respectively, one cannot exclude the influence of a possible bias. Indeed, there was an underestimation of the hand territory lying in the rostral bank of the central sulcus, where a penetration all the way down the bank was represented by a single point on the surface map of Figure $2 \mathrm{~A}$. For this reason, as done and explained in the next paragraph, one may consider not only one ICMS site for each penetration (corresponding to the lowest threshold), but all ICMS sites tested. As reported in Figure 2B for Mk1, 67 ICMS sites generated distal (hand) movements pre-lesion, a number dropping to 21 sites post-lesion, thus representing a drop of $69 \%$. For Mk2, the number of ICMS sites eliciting distal movements dropped from 74 to 2 after the lesion, corresponding to a decrease of $97 \%$. These values reflecting the decrease of hand ICMS sites, particularly for Mk2, thus support the notion of underestimation of the territory buried in the rostral bank of the central sulcus on the surface map such as in Figure 2A. 


\subsubsection{Comparison of thresholds}

The surface of the M1 hand area post-lesion dramatically decreased after the lesion (Fig. 2A) and the number of sites eliciting digit movements post-lesion was also reduced. They were replaced by sites eliciting forelimb proximal or face movements or, in some cases, the sites became non micro-excitable. These observations were expected because the lesion reduced greatly or completely the number of CS neurons accessing directly to the motoneurones placed caudally to the lesion (i.e. motoneurones innervating the finger muscles), but preserved access to the motoneurones placed more rostrally via collaterals. As a consequence of the diminished access to finger motoneurones, one would predict that a higher stimulation current would be necessary to elicit finger movements than before the lesion. To test this prediction, we compared the ICMS thresholds required to elicit movements from stimulation at the same stereotaxic points before and after the lesion, in the later case when the behavioral score reached the plateau. In contrast to Figure $2 \mathrm{~A}$ where only the best ICMS site along each electrode penetration was represented, several sites of stimulation were considered along each track for the ICMS threshold comparison (Fig. 2B). Based on the body territories affected, ICMS sites were grouped in three sets (Fig. 2B): distal (movements of the digits), proximal (movements of the wrist, elbow or shoulder) and face. A mean value of thresholds (with the standard deviation) has been obtained for these three groups, pre- and post-lesion (Fig. 2B). Surprisingly, for both animals, the threshold stimulation current to elicit either finger, proximal forelimb or face movements remained comparable pre- and post-lesion (no statistically significant difference; Mann Whitney U-test, $p>0.05)$.

\subsection{Progressive changes of ICMS effects during the recovery period}

The ICMS data shown in Fig. 2A, limited to two time points, do not address the question of the time course of the plastic changes post-lesion and to what extent the temporal evolution of plastic changes parallels the time course of behavioral recovery. To address this question, we conducted ICMS experiments repetitively at short time intervals during the few weeks following the lesion. Daily ICMS sessions on alert monkeys were, however, limited to 30-60 minutes, allowing a 
number of electrode penetrations ranging from 3 to 6 , in general. Thus, a complete mapping of the hand area in M1 (like in Fig. 2A) requires 10 to 20 daily sessions on each hemisphere. Therefore, a complete mapping of the hand area at weekly intervals post-lesion during the recovery was not undertaken, also to avoid an additional deficit to that initially induced by the cervical cord lesion. For these reasons, a limited number of electrode track locations were selected (see Fig. 2A), where ICMS experiments were systematically repeated approximately every 7 days during the period of functional recovery (days 2-6 to days 40-48 post-lesion; Figs. 3 and 4). Four electrode track positions were selected for the repetitive ICMS protocol, as illustrated in Fig. 3 for Mk1. Three of the tracks $(\# 1,2,3)$ belonged to the hand territory while ICMS along the fourth track elicited twitches of face muscles ("F").

A repetition of ICMS at the same locations two days post-lesion yielded quite different results in the hand area. In the electrode tracks \#1 and \#3, ICMS did not elicit movements of the fingers any more, but produced movements of more proximal muscles or failed to induce any observable movement (Fig. 3, second column from the left). Along the electrode track \#2 (Fig. 3), the number of sites where ICMS elicited finger movements decreased dramatically (seven before the lesion and only one post-lesion). In addition, the finger movements produced at the latter site were obtained at a higher current intensity than that necessary to elicit movements of other territories (wrist). Note that ICMS conducted along the electrode track initially performed in the face area of M1 did not show any significant change of representation during the entire recovery period (Fig. 3, bottom line).

Eight days post-lesion, a limited re-appearance of finger movements in the hand area occurred. In electrode track \# 2, four points along the track elicited finger movements, as compared to one point 7 days before. Again, these ICMS sites were less sensitive than other sites triggering movements of other body territories (wrist). One site where ICMS elicited finger movements reappeared at that time point along electrode track \#3 (black star), but once more this was not the site of maximal excitability. Finger movements were observed to re-appear in electrode track \#1 as well, after 22 days. ICMS sessions performed at day 22 or more post-lesion confirmed this tendency of a re-appearance of ICMS sites addressing hand muscles. The immediate post-lesion 
dramatic loss of virtually all sites producing finger movements pre-lesion was replaced by a progressive, incomplete, re-establishment of sites where finger movements occurred as a result of ICMS (black ticks pointed by stars in the three top lines, days 22 to 40 of Figure 3). Along electrode track \#2, stimulation of the site of lowest threshold elicited finger movements, thus re-establishing a hand representation post-lesion.

Consistent progressive ICMS data were observed in Mk2, as shown by repetitive ICMS sessions performed during recovery along two electrode penetrations (Fig. 4). All sites where ICMS elicited finger movements were replaced, at six and thirteen days post-lesion, by wrist movements. Later on in track \#1, in parallel to the progressive functional recovery, a few sites where finger movements could be elicited by ICMS re-appeared (27, 41 and 48 days post-lesion). However, these few re-established hand points did not correspond to the lowest threshold along the electrode penetration on the long term and therefore the location of the electrode penetration on the surface map does not correspond to a hand representation (Fig. 2A). In this animal (Mk2), we also observed electrode penetrations in the pre-lesion hand area along which there was no reappearance of sites eliciting hand movements (track \#2 in Fig. 4), after the elimination of such ICMS sites immediately after lesion. These observations indicate that the phenomenon of map plasticity in parallel to the recovery is limited and its occurrence appears to be related to the size of the lesion. Indeed, substantially more ICMS digit sites reappeared post-lesion in Mk1 subjected to a smaller lesion than in Mk2 with a larger cervical cord lesion.

\subsection{Time course of ICMS changes and time course of behavioral recovery}

The progressive ICMS changes observed in Mk1 during the few weeks post-lesion (Fig. 3) indicate that sites eliciting finger movements, which nearly completely disappeared as a result of the lesion, re-appeared in a stable manner in the 3 tested penetrations after 15-22 days. This time point was correlated with the behavioral recovery curve of the same monkey (Mk1), showing that a substantial degree of recovery of $50 \%$ was reached after about 2 weeks. In Mk2, the recovery of manual dexterity was clearly less prominent and slower, with a small, but consistent, ability to again perform the grasping movement from 25 days post-lesion. As seen in the progressive ICMS 
assessment during the few weeks post-lesion (Fig. 4), the first time point at which sites eliciting finger movements re-appeared was 27 days. Although these data are limited to two monkeys and to a limited set of ICMS penetrations, there is some evidence for a correlation between the behavioral recovery and the progressive plastic changes of the hand representation in the motor cortex contralateral to the lesion.

\subsection{Does the progressively re-established hand area contribute to the recovery?}

To address this question, reversible inactivation sessions of $M 1$ in the ipsilateral or contralateral hemisphere were performed 3 and 5 months post-lesion in Mk1 and Mk2, respectively. In a typical inactivation session obtained by infusion of muscimol, the manual dexterity task was initially performed just before the muscimol infusion and repeated $20-30$ minutes after completion of the muscimol injections - a time point at which its effect is well established. A pre- and post-inactivation manual dexterity score was thus determined for each hand. Before infusion of muscimol, the behavioral score of the affected hand was that corresponding to the plateau of incomplete recovery (Fig. 1B). In both monkeys, a reversible inactivation of the ipsilateral M1 did not affect noticeably the recovered manual dexterity score of the hand homolateral to the cervical lesion, which thus remained at the recovery plateau level. In sharp contrast, a reversible inactivation of the contralateral M1, including the re-established hand area, led to a total inability to make coordinated finger movements as reflected by a manual dexterity score dropping to zero (the same level as immediately after the lesion). This second observation demonstrates that the contralateral M1 contributes substantially to the incomplete recovery, in contrast to the ipsilateral hemisphere. Recovery from inactivation using muscimol is slow (several hours) and therefore could not be tested within the same inactivation session. However, the day after, the animal had fully recovered its manual dexterity from before the inactivation session. 


\section{Discussion}

The present study aimed at assessing the effect of partial spinal cord lesions, as well as the amount and time course of spontaneous recovery that can take place post-lesion. The understanding of such mechanisms is of strategic significance in the context of ongoing work leading to attempts to repair human spinal cord injury. In particular, such knowledge derived from the present non-human primate model is crucial, because of the potential difficulties in translating work in the rat model of spinal cord injury to the human condition. In macaque monkeys, we used a skilled hand task to confirm that some spontaneous recovery can and does occur following a subtotal cervical hemi-section. Quantitative behavioral measures also demonstrate that the recovery is limited and may to some extent depend on compensatory strategies. Whatever the precise strategies are, reversible inactivation experiments clearly demonstrated the contribution of the contralesional primary motor cortex to the recovered function. These results are of significance in attempting to assess the impact of any interventions that might further improve recovery of function after spinal cord injury.

\subsection{Technical considerations}

Data in Figure 2 show clear changes of cortical representations in M1 when comparing ICMS maps established before and after cervical hemi-section. In addition, the representation of body territories affected by the lesion (digits) exhibited post-lesion progressive changes, in parallel to the time course of recovery. These observations are based on the comparison of ICMS data derived from penetrations performed at the same cortical location at various time points, for instance before and after the lesion. However, one may question the intrinsic variability of the ICMS method. In other words, are ICMS changes attributed to a lesion clearly more prominent than variations observed along repetitive penetrations performed at the same location in an intact animal? To address this crucial question, a few ICMS penetrations at the same site were repeated during the pre-lesion period, while the monkeys were intact. Overall, in the two monkeys, six and two ICMS electrode penetrations were repeated either twice or even three times, respectively. The time interval between two repetitions ranged from 2 to 71 days (median value: 12 days). While one 
electrode penetration exhibited activation of different body territories at two time points (digits replaced by face), the 7 other repetitions of electrode penetrations were found to be very reproducible. More precisely, we observed similar body territories activated at lowest threshold, comparable sequences of movements observed along the track at various depths and consistent relative amplitudes of current needed to elicit the observed movements. These data argue for a good reproducibility of the ICMS method and, therefore, one can conclude that the ICMS plastic changes as described in Figures 2, 3 and 4 are substantial and reflect a true plasticity induced by the cervical lesion. Furthermore, such stability of ICMS data observed for electrode penetrations repeated up to three times is consistent with the notion that our protocol of stimulation did not generate damage of the cortical tissue due to over-penetration. This conclusion is in line with our previous work $[25,36]$, where no deficit of manual dexterity was observed when the hand area was mapped using the same ICMS protocol while the animal was intact.

\subsection{Pathways affected by the lesion}

The position and extent of the lesion at the transition between the seventh and eighth cervical segments (Fig. 1A) indicates that most of the drive exerted by the motor cortex on finger muscle motoneurones was eliminated. In the macaque monkey, the location of the CS axons at cervical level, forming three different fascicles, is well established $[2,7,21,35]$. More quantitatively, as shown in a separate anatomical study [37], unilateral injection of the anterograde tracer BDA in the hand area of M1 resulted in the following distribution of CS axons at cervical level. Nearly $90 \%$ of the CS axons originating from the injected hemisphere decussate, while the rest (about 10\%) does not decussate, running along the ipsilateral dorso-lateral fasciculus, although a few CS axons $(1-2 \%)$ travel along the ipsilateral ventro-medial fasciculus. Most of the left dorsolateral fasciculus was thus sectioned in both animals (Fig. 1A), although a small proportion may have been spared in Mk1. One may also not totally exclude the possibility that a few intraspinal collaterals of stem CS axons can travel for some distance within the spinal grey matter [23], and thus could still influence motoneurones in 1 or even 2 segments below the funicular lesion. Despite of the absence of direct quantitative measures, it seems safe to estimate that $80-90 \%$ of the drive normally provided 
directly by the CS system to spinal segments caudal to the lesion (and including that of the spared uncrossed projections) has been eliminated. In addition, the drive provided by other descending systems (particularly that of the rubrospinal projection) has been partially diminished as well.

The above mentioned distribution of CS axons at cervical level in the three components of the CS tract is consistent with the classical view of a vast majority (about $90 \%$ ) of decussated CS axons travelling in the dorsolateral funiculus. However, the higher proportion of undecussated CS axons found in the ipsilateral dorsolateral funiculus than in the ipsilateral ventral funiculus contrasts with the classical view of more numerous undecussated CS axons in the ventral funiculus, as seen in macaca mulata or chimpanzee [21]. This discrepancy can be explained by different zones of origin of the CS projection in M1, precisely restricted to the hand area in our study. In addition, the classical view is based on observations derived from tracing methods (anterograde degeneration, autoradiography), which do not allow identification and precise counts of individual CS axons, in contrast to BDA, suggesting that the latter method led to more accurate counts.

The clear loss of ICMS responsive sites in M1 during 1-2 weeks after the lesion (see Figs. 3 and 4) was interpreted primarily as the consequence of the transection of the CS axons mediating the control of M1 onto the corresponding distal muscle motoneurones, before some re-organization took place. However, one may question to what extent such observation relates to what has been referred to as "spinal shock", although the physio-pathology of this phenomenon remains largely unknown. We believe that both the small size of the spinal lesion and its greater impact on the white than gray matter is likely to generate a less prominent "spinal shock" than a larger spinal lesion as often observed in human subjects. In addition, the muscles supporting the finger movements of the affected hand were not flaccid. These considerations thus argue against a "spinal" phenomenon and rather support the notion that the loss of ICMS response is indeed due to lack of CS input.

\subsection{Extent and time course of functional recovery}

After subtotal hemi-section of the cervical cord at C7/C8 level, the time course of recovery of manual dexterity observed in the present work (about 50 days) is generally consistent with the 60- 
90 days reported following complete unilateral section of the cervical cord at C3 level in juvenile monkeys [8]. However, the latter authors reported persisting deficit, in line with our data demonstrating that the recovery is incomplete. The recovered grip movement can differ from the original pre-lesion pattern of movement, indicating that rehabilitation is, at least partly, based on the development of compensatory movements, as previously reported after cortical lesion [6] or cervical cord lesion [8]. In the present case, the prehension of pellets was executed more slowly and the monkeys did not use the typical opposition of thumb and index finger, indicating that coordination of the fingers was affected. In one animal (Mk2), the thumb was even largely unused, being kept in a flexed position. As a result, the pellet was grasped essentially using the index finger by pushing the pellet towards the dorsal part of the flexed thumb or towards the palm of the hand.

Previous data derived from cortical lesions have shown that an intense rehabilitative training (frequent use of the impaired hand) improves the recovery and leads to a re-expansion of the digit representation [32]. Such intense rehabilitative training was not considered here because our goal was to establish a baseline of "spontaneous" recovery for the cervical cord hemi-section model. Nevertheless, we cannot exclude that the daily manual dexterity testing ("modified Brinkman board" task) represented a mild rehabilitative training, which presumably led to a slightly better recovery than that obtained if the monkey would have been tested only after several months. The work of Nudo and collaborators $[30,31,32]$ thus suggests that the decrease of the hand representation in the contralesional cortex would have been less dramatic if a more intense use of the affected hand had occurred.

\subsection{Mechanisms of functional recovery}

The ICMS experiments confirm that a spinal cord lesion modifies cortical maps, as previously reported in monkeys $[16,17]$ and human subjects $[9,10,26,27,29,33]$. However, in human subjects, the pre-lesion cortical map was not available and the post-lesion data were compared with a group of normal subjects, thus introducing a large variability in the data. In contrast, the monkey model as used here allows a comparison of the cortical map pre- and post-lesion. The present study thus provides evidence, for the first time, that the time course of recovery after hemi-section of the 
cervical cord parallels the progressive changes taking place in the somatotopic map in the motor cortex contralateral to the lesion. Although the parallel time courses of recovery and cortical plastic changes do not prove a causal relationship between the two phenomena, reversible inactivation of the reorganized motor cortex several months after the lesion abolished the manual dexterity regained during the recovery. In contrast, reversible inactivation of the motor cortex homolateral to the cervical lesion did not affect the recovered behavioral score. Consequently, a possible mechanism of recovery involving decussated CS axons originating from the ipsilesional cortex, sprouting in the intact spinal side before regaining control of deprived motoneurones by re-crossing the midline below the lesion is unlikely. These observations support the notion that, although substantially diminished in size, the post-lesion hand territory in the contralesional M1 plays a crucial role in the partial rehabilitation.

The pathways through which the reorganized hand area in the contralesional M1 regains partial control of the muscles of the affected hand remains unclear. Previous data [7] and preliminary observations from our laboratory indicate that there is no regeneration of the transected CS axons. Furthermore, we have evidence that most axotomized CS neurons in the contralateral M1 do not degenerate [37], but rather exhibit shrinkage of their soma. A mechanism of compensatory sprouting can come into play in the form of a re-innervation of the denervated motoneurones by undecussated CS axons originating from the contralesional M1 and crossing the midline at segmental level below the lesion. The time course of reorganization in M1 may thus reflect the time taken for such compensatory sprouting. One may also argue that the post-lesion control exerted by the contralesional M1 may be undertaken by decussated CS axons spared by the lesion. Such interpretation is unlikely, particularly for Mk2, considering the location and extent of the lesion (Fig. 1A) and, in addition, such control would be effective immediately after the lesion. Indeed, the delayed recovery and progressive re-arrangement of the hand representation in M1 over several weeks are rather supportive of slow mechanisms of compensation via reorganized pathways, such as the undecussated CS axons running in the contralesional dorso-lateral and/or ventral lateral funiculi. Such a scenario of recovery involving minor CS tracts not affected by the lesion has been verified in the rat. Spontaneous sprouting of the CS ventral funiculus after lesion at 
cervical level of the main CS tract in the dorsal funiculus was found to parallel recovery [38]. Elegantly, these authors observed that a combined lesion of both the CS dorsal and ventral funiculi eliminated sprouting and recovery in the rat. Based on these rat data, one may speculate that a larger cervical lesion at C7/C8 in the monkey (affecting the uncrossed CS tracts) would result in a slower and less prominent recovery, as compared to the monkeys of the present study. One should also not disregard the possibility of compensatory sprouting taking place immediately above the lesion by forming new intraspinal circuits (e.g. $[3,5,14])$ or at higher levels. For instance, both CS neurons not affected by the lesion and/or axotomized CS neurons may well sprout in the brainstem in order to recruit other descending pathways such as the rubrospinal or reticulospinal projection systems [21]. However, in adult rats subjected to pyramidotomy, such post-lesional sprouting in the brainstem was substantial only after blockade of myelin-associated neurite growth inhibitors [40]. This scenario of recovery via the brainstem is consistent with the observation of significant sprouting of the rubrospinal projection in the spinal cord, after pyramidotomy and blockade of myelin-associated neurite growth inhibitors [34]. Also consistent with such a scenario, in the contusive spinal cord injury model in the rat, a collateralization and penetration of reticulospinal fibers in the lesion matrix was observed [14]. In the present monkey study, the rubrospinal projection was partially (Mk1) or substantially (Mk2) affected by the lesion, in contrast to the ventromedially located reticulospinal tract largely unaffected by the cervical hemi-section. Therefore, the cortico-reticular and reticulospinal projections may have contributed to the observed behavioral recovery, more than the cortico-rubral and rubrospinal projections. Irrespective of the precise scenario of remodeling, it seems that the preservation of a small contingent of intact CS axons (the undecussated ones) is a necessary condition for the plastic changes in motor maps as observed here in Figs. 2, 3 and 4. Indeed, it was reported that ICMS did not elicit motor responses following a complete pyramidotomy [28].

The re-routing of the control of hand muscles via remodeled connections appears very efficient functionally, as evidenced by the comparable ICMS thresholds before and after the lesion (Fig. 2B). It should be noted that the comparison for distal movements is problematic in Mk2, since only two sites eliciting digit movements were left at the time point at which the ICMS map was 
established post-lesion. Nevertheless, based on the data in Mk1, it appears that the ICMS sites eliciting movements of the digits post-lesion did not exhibit elevated threshold. An absence of elevation of ICMS thresholds post-lesion was also observed for other movements, namely of face and proximal forelimb muscles (Fig. 2B). The observation that ICMS thresholds were not significantly higher post-lesion (Fig. 2B) may be explained, at least in part, by considering that threshold measurements were performed at a time period after the recovery had reached a plateau. One cannot thus exclude that ICMS thresholds were elevated during the recovery period.

The present data and previous studies in the rat [38] argue for a significant role played in the recovery by undecussated axons originating from the contralesional motor cortex and not affected by the lesion. However, further anatomical data are needed, derived from injection of an anterograde tracer in the contralesional motor cortex, in order to identify at which level undecussated CS axons sprout in order to compensate for the deficits induced by the cervical lesion. Such evidence of compensatory sprouting may be difficult to establish because it may well be present in a relatively limited number of CS axons and therefore difficult to detect while comparing with a normal animal. 


\section{References}

[1] M. Aoki and S. Mori, Recovery of hindlimb movement elicited by motor cortical stimulation after spinal hemisection in monkeys. In M. Ito (Ed.), Integrative control functions of the brain, Elsevier, Amsterdam, 1979, pp. 152-154.

[2] J. Armand, E. Olivier, S.A. Edgley and R.N. Lemon, Postnatal development of corticospinal projections from motor cortex to the cervical enlargement in the Macaque monkey, J. Neurosci. 17 (1997) 251-266.

[3] F.M. Bareyre, M. Kerschensteiner, O. Raineteau, T.C. Mettenleiter, O. Weinmann and M.E. Schwab, The injured spinal cord spontaneously forms a new intraspinal circuit in adult rats. Nat. Neurosci. 7 (2004) 269277.

[4] D. Denny-Brown, The cerebral control of movements. Liverpool University Press, Liverpool, 1966.

[5] T.W. Ford, C.W. Vaughan and P.A. Kirkwood, Changes in the distribution of synaptic potentials from bulbospinal neurones following axotomy in cat thoracic spinal cord. J. Physiol. 524 (2000) 163-178.

[6] K.M. Friel and R.J. Nudo, Recovery of motor function after focal cortical injury in primates: compensatory movement patterns used during rehabilitative training, Somatosens. Mot. Res. 15 (1998) 173-189.

[7] M.P. Galea and I. Darian-Smith, Corticospinal projection patterns following unilateral section of the cervical spinal cord in the newborn and juvenile macaque monkey, J. Comp. Neurol. 381 (1997a) 282-306.

[8] M.P. Galea and I. Darian-Smith, Manual dexterity and corticospinal connectivity following unilateral section of the cervical spinal cord in the macaque monkey, J. Comp. Neurol. 381 (1997b) 307-319.

[9] J.B. Green, E. Sora, Y. Bialy, A. Ricamato and R.W. Thatcher, Cortical sensorimotor reorganization after spinal cord injury - An electroencephalographic study, Neurology 50 (1998) 1115-1121.

[10] J.B. Green, E. Sora, Y. Bialy, A. Ricamato and R.W. Thatcher, Cortical motor reorganization after paraplegia - An EEG study, Neurology 53 (1999) 736-743.

[11] M.C. Hepp-Reymond, E. Trouche and M. Wiesendanger, Effects of unilateral and bilateral pyramidotomy on a conditioned rapid precision grip in monkeys (Macaca fascicularis), Exp. Brain Res. 21 (1974) 519-527.

[12] M.C. Hepp-Reymond and M. Wiesendanger, Pyramidotomy in monkeys: effect on force and speed of a conditioned precision grip, Brain Res. 36 (1972) 117-131.

[13] M.C. Hepp-Reymond, Lésions expérimentales dans le système nerveux central. Pädiat. Fortbildk. Praxis 53 (1982) 160-177.

[14] C.E. Hill, M.S. Beattie and J.C. Bresnahan, Degeneration and sprouting of identified descending supraspinal axons after contusive spinal cord injury in the rat. Exp. Neurol. 171 (2001) 153-169. 
[15] G. Holmes and W.P. May, On the exact origin of the pyramidal tracts in man and other mammals. Brain 32 (1909) 1-43.

[16] N. Jain, K.C. Catania and J.H. Kaas, Deactivation and reactivation of somatosensory cortex after dorsal spinal cord injury, Nature 386 (1997) 495-498.

[17] N. Jain, S.L. Florence and J.H. Kaas, Reorganization of somatosensory cortex after nerve and spinal cord injury. News Physiol. Sci. 13 (1998) 143-149.

[18] A.B. Jenny and J. Inukai, Principles of motor organization of the monkey cervical spinal cord. J. Neurosci. 3 (1983) 567-575.

[19] I. Kermadi, Y. Liu, A. Tempini and E.M. Rouiller, Effects of reversible inactivation of the supplementary motor area (SMA) on unimanual grasp and bimanual pull and grasp performance in monkeys, Somatosens. Mot. Res. 14 (1997) 268-280.

[20] I. Kermadi, Y. Liu, A. Tempini, E. Calciati and E.M. Rouiller, Neuronal activity in the primate supplementary motor area and the primary motor cortex in relation to spatio-temporal bimanual coordination, Somatosens. and Mot. Res. 15 (1998) 287-308.

[21] H.G.J.M. Kuypers, Anatomy of descending pathways. In Brooks VB (Ed.) Handbook of Physiology (The Nervous System), vol. II, part 1 (1981). Bethesda, MD, Am. Physiol. Soc. pps. 597-666.

[22] D.G. Lawrence and H.G.J.M. Kuypers, The functional organization of the motor system. I. The effects of bilateral pyramidal lesions, Brain 91 (1968) 1-14.

[23] D.G. Lawrence, R. Porter and S.J. Redman, Corticomotoneuronal synapses in the monkey:light microscopic localization upon motoneurons of intrinsic muscles of the hand, J. Comp. Neurol. 232 (1985) 499-510.

[24] J. Liu, A. Morel, T. Wannier and E.M. Rouiller, Origins of callosal projections to the supplementary motor area (SMA): a direct comparison between pre-SMA and SMA-proper in macaque monkeys, J. Comp. Neurol. 443 (2002) 71-85.

[25] Y. Liu and E.M. Rouiller, Mechanisms of recovery of dexterity following unilateral lesion of the sensorimotor cortex in adult monkeys, Exp. Brain Res. 128 (1999) 149-159.

[26] M. Lotze, U. Laubis-Herrmann, H. Topka, M. Erb and W. Grodd, Reorganization in the primary motor cortex after spinal cord injury - A functional Magnetic Resonance (fMRI) Study, Restor. Neurol. Neurosci. 14 (1999) 183-187.

[27] R.S. Marshall, G.M. Perera, R.M. Lazar, J.W. Krakauer, R.C. Constantine and R.L. DeLaPaz, Evolution of cortical activation during recovery from corticospinal tract infarction, Stroke 31 (2000) 656-661. 
[28] A.R. Mitz and D.R. Humphrey, Intracortical stimulation in pyramidotomized monkeys, Neurosci. Lett. 64 (1986) 59-64.

[29] G. Nelles, G. Spiekermann, M. Jueptner, G. Leonhardt, S. Müller, H. Gerhard and H.C. Diener, Reorganization of sensory and motor systems in hemiplegic stroke patients - A positron emission tomography study, Stroke 30 (1999) 1510-1516.

[30] R.J. Nudo, Recovery after damage to motor cortical areas, Curr. Opin. Neurobiol. 9 (1999) 740-747.

[31] R.J. Nudo and G.W. Milliken, Reorganization of movement representations in primary motor cortex following focal ischemic infarcts in adult squirrel monkeys, J. Neurophysiol. 75 (1996) 2144-2149.

[32] R.J. Nudo, B.M. Wise, F. SiFuentes and G.W. Milliken, Neural substrates for the effects of rehabilitative training on motor recovery after ischemic infarct, Science 272 (1996) 1791-1794.

[33] B.K. Puri, H.C. Smith, I.J. Cox, J. Sargentoni, G. Savic, D.W. Maskill, H.L. Frankel, P.H. Ellaway and N.J. Davey, The human motor cortex after incomplete spinal cord injury: an investigation using proton magnetic resonance spectroscopy, J. Neurol. Neurosurg. Psychiatry 65 (1998) 748-754.

[34] O. Raineteau, K. Fouad, F.M. Bareyre and M.E. Schwab, Reorganization of descending motor tracts in the rat spinal cord. Europ. J. Neurosci. 16 (2002) 1761-1771.

[35] E.M. Rouiller, V. Moret, J. Tanné and D. Boussaoud, Evidence for direct connections between the hand region of the supplementary motor area and cervical motoneurons in the macaque monkey, Eur. J. Neurosci. 8 (1996) 1055-1059.

[36] E.M. Rouiller, X.H. Yu, V. Moret, A. Tempini, M. Wiesendanger and F. Liang, Dexterity in adult monkeys following early lesion of the motor cortical hand area: the role of cortex adjacent to the lesion, Eur. J. Neurosci. 10 (1998) 729-740.

[37] T. Wannier, E. Schmidlin, J. Bloch and E.M. Rouiller, A unilateral section of the corticospinal tract at cervical level in primate does not lead to measurable cell loss in motor cortex (submitted for publication).

[38] N. Weidner, A. Ner, N. Salimi and M.H. Tuszynski, Spontaneous corticospinal axonal plasticity and functional recovery after adult central nervous system injury, Proc. Natl. Acad. Sci. U S A 98 (2001) 35133518.

[39] C. Xerri, M.M. Merzenich, B.E. Peterson and W. Jenkins, Plasticity of primary somatosensory cortex paralleling sensorimotor skill recovery from stroke in adult monkeys, J. Neurophysiol. 79 (1998) 2119-2148.

[40] W.J. Z'graggen, G.A.S Metz, G.L. Kartje, M. Thallmair and M.E. Schwab, Functional recovery and enhanced corticofugal plasticity after unilateral pyramidal tract lesion and blockade of myelin-associated neurite growth inhibitors in adult rats. J. Neurosci. 18 (1998) 4744-4757. 


\section{Legend to Figures}

\section{Figure 1}

A: Location and extent of the unilateral lesion (grey area), performed at C7/C8 in Mk1 and Mk2, shown on a reconstructed frontal view of cervical cord. Scale bar $=2 \mathrm{~mm}$.

B: Behavioral scores in Mk2 reflecting manual dexterity for the hand ipsilateral (top panel), and contralateral (bottom panel), to the cervical lesion, before (days -300 to 0 ) and after (days 0-200) the cervical lesion in Mk2, performed at day 0 . Manual dexterity was assessed using the so-called "modified Brinkman board" test (see methods). The behavioral score was defined as the number of food pieces successfully retrieved within 1 min, using the precision grip movement (opposition of thumb and index finger).

\section{Figure 2}

A: Somatotopic map in the motor cortex on the right (contralesional) hemisphere in the region of the hand area, before (left column) and after (right column) the cervical lesion. The pre-lesion maps were established by daily ICMS sessions conducted during the 2 months preceding the lesion. The post-lesion maps were derived from daily ICMS sessions starting 2 and 4.5 months after the lesion in Mk1 and Mk2, respectively and lasted about 2 months each. The data are presented on a surface view of the brain. Each symbol represents the location of penetration with an electrode for ICMS. The ICMS data given for each symbol is representative for the site of stimulation where the effect was observed at the lowest current intensity along the considered electrode track. The letter next to each symbol indicates the body territory activated at threshold for each track (see letter codes in the bottom right). The current intensity at threshold (in $\mu \mathrm{A}$ ) is indicated by the size of the symbols (see bottom left). Hand area(s), outlined by a solid line, was defined as a cortical region where ICMS at lowest threshold elicits movements of the fingers (electrode tracks represented by diamonds). The map is given for Mk1 (top two panels) and Mk2 (bottom two panels), as established by ICMS before (left two panels) and after lesion (right two panels). Symbol $\mathrm{X}$ means that ICMS did not elicit any visible movement of muscles. Symbols in 
grey are for sites belonging to the hand area before lesion, which became part of other territories post-lesion. The grid in the background indicates steps of $1 \mathrm{~mm}$. In the pre-lesion maps (left column), symbols outside the hand area with a small white rectangle indicate electrode penetrations along which hand movements were obtained, but not at the lowest current producing ICMS effect. The position of the tracks in which ICMS was repeated on a weekly basis during the period of recovery are pointed by numbers (1-4 for Mk1, see Fig. 3; 1-2 for MK2, see Fig. 4). Syringes point to ICMS sites where muscimol was infused in order to inactivate M1 (see text). In order to make sure that the entire "post-lesion" hand representation was reversibly inactivated, the sites of infusion of muscimol were rather selected based on the "pre-lesion" map, exhibiting a larger hand representation than post-lesion. On the pre-lesion maps (left column), the approximate positions of the central (CE) and arcuate (AR) sulci are indicated by dashed lines.

B: For M1 contralateral to the lesion, the bars indicate the average ICMS current at threshold with standard deviations to elicit movements of forelimb "distal" muscles (fingers), forelimb "proximal" muscles (wrist, elbow, shoulder) or "face" muscles. The average values are derived from all responsive ICMS sites cumulated before ("pre-") and after ("post-") lesion, separately for the two monkeys. The pre-lesion ICMS threshold data were collected during the 2 months preceding the lesion, whereas the post-lesion ICMS threshold data were collected during a period of 2 months, starting 2 and 4.5 months after the lesion in Mk1 and Mk2, respectively. The number of ICMS sites considered to establish these data (given in the bottom of each bin) was lower post-lesion than pre-lesion because some ICMS sites became non-microexcitable post-lesion. Note that the ICMS thresholds were not significantly different pre- and post-lesion.

\section{Figure 3}

In order to establish the time course and extent of progressive plastic changes taking place during the recovery period, four cortical sites were penetrated repetitively in $\mathrm{M} 1$ contralateral to the cervical lesion, and ICMS was applied at the same sites on a period of 40 days post-lesion (abscissa). The data obtained for four electrode penetrations are presented here for Mk1 (the vertical bars with horizontal ticks numbered 1 to 4 ). The top of the four vertical bars corresponds to 
the surface of the dura. Then, ticks correspond to progressively deeper ICMS sites, separated from each other by $1 \mathrm{~mm}$. The four bars on the left of the vertical dashed line are ICMS data before

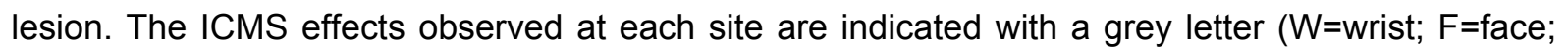
$E=E l b o w ; S=S h o u l d e r ; T=T r u n k$ ) or with a black star in case of movements of the fingers. At each site, on a scale at the right of each electrode penetration, the threshold at which the ICMS effect was observed is indicated (between 0 and $80 \mu \mathrm{A}$ ). The depth at which the current was the lowest along the corresponding electrode penetration is indicated by an arrow. Non-microexcitable sites are indicated by $\mathrm{X}$ symbol.

\section{$\underline{\text { Figure } 4}$}

Progressive plastic changes taking place along two electrode penetrations in Mk2 during the period of recovery of manual dexterity. Same conventions as in Figure 3. 

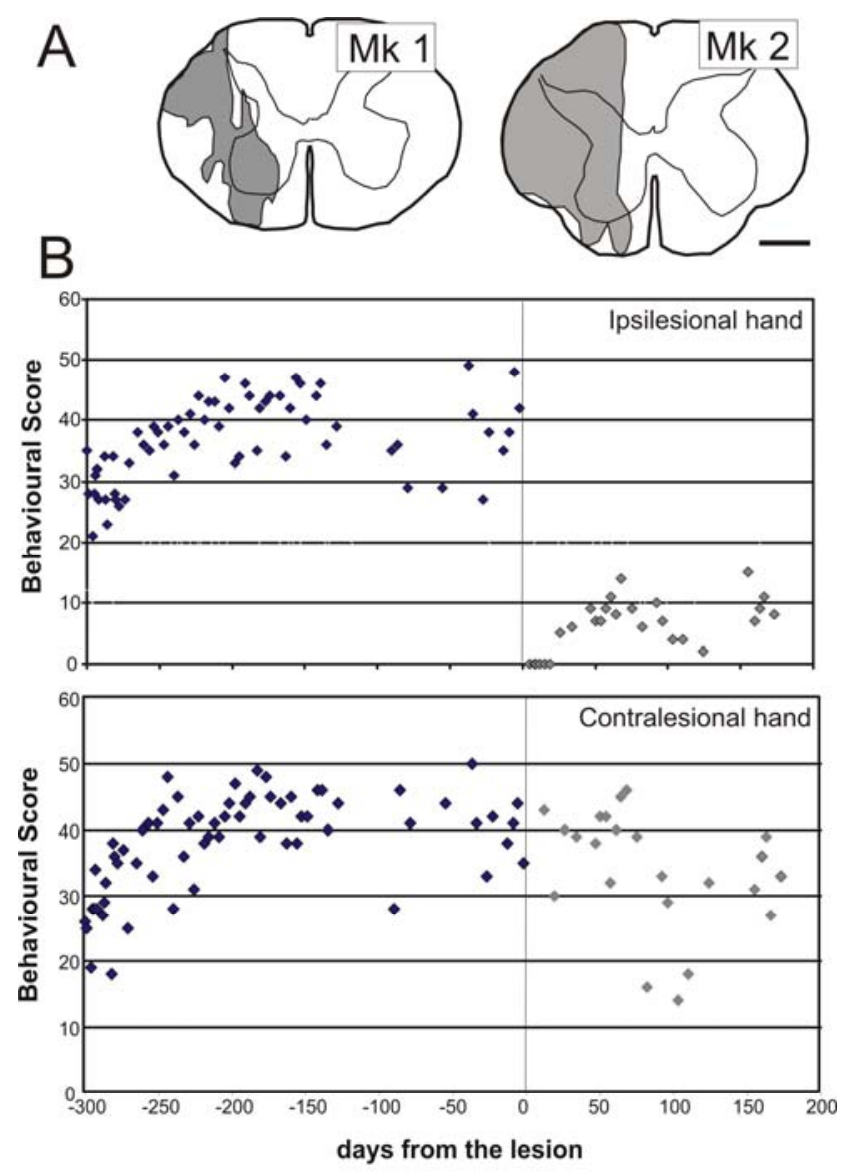

Fig. 1 (Schmidlin et al.) 
$\underline{A}$
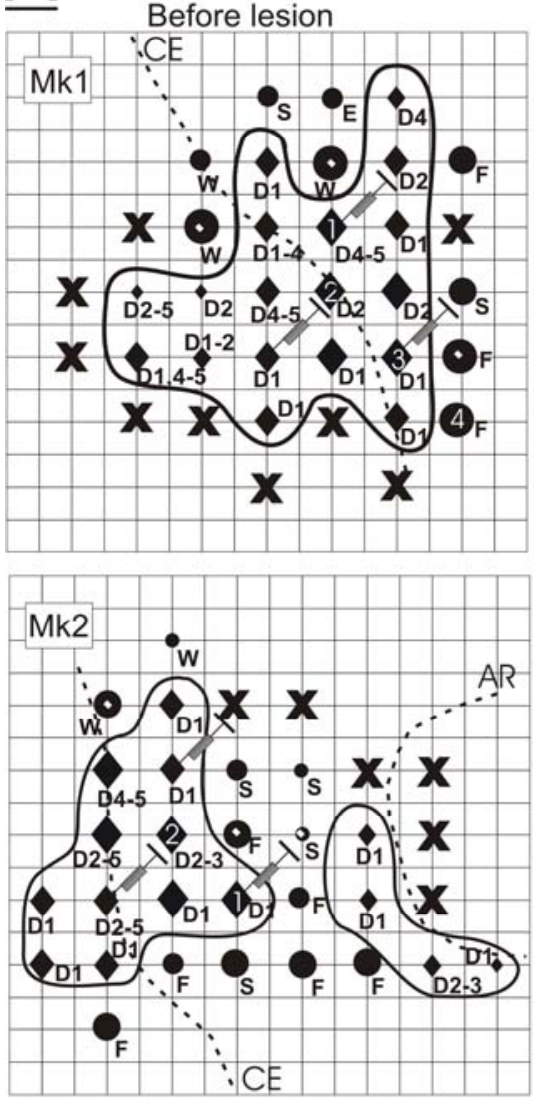

\begin{tabular}{c|c|c|c|c|c|c|} 
Threshold current $($ in $\mu \mathrm{A})$ & $<=10$ & $11-30$ & $31-50$ & $51-70$ & $71-90$ \\
\hline Digit movements & & - & $\bullet$ & $\bullet$ & $\bullet$ \\
\hline Other movements & & & $\bullet$ & $\bullet$ & $\bullet$
\end{tabular}

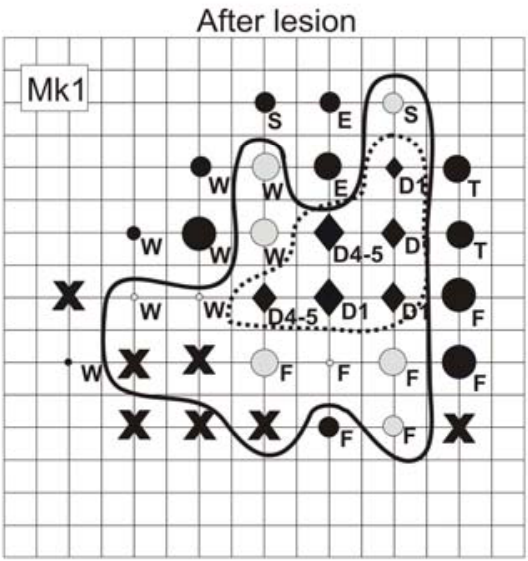

$\underline{B}$

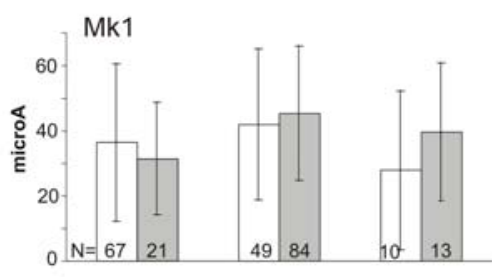

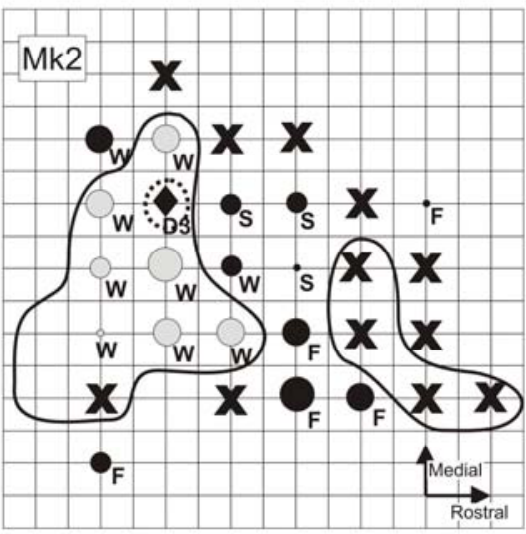

$\mathrm{D}=$ Digits

$\lambda=$ Muscimol injection site

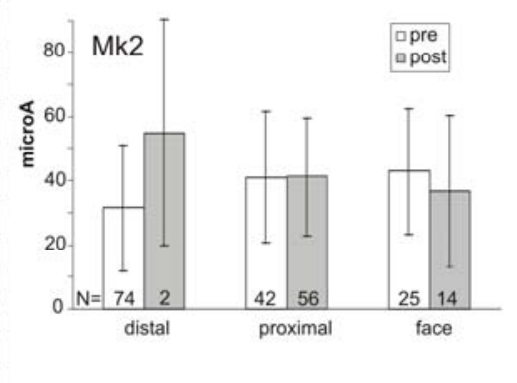

Fig. 2 (Schmidlin et al.) 


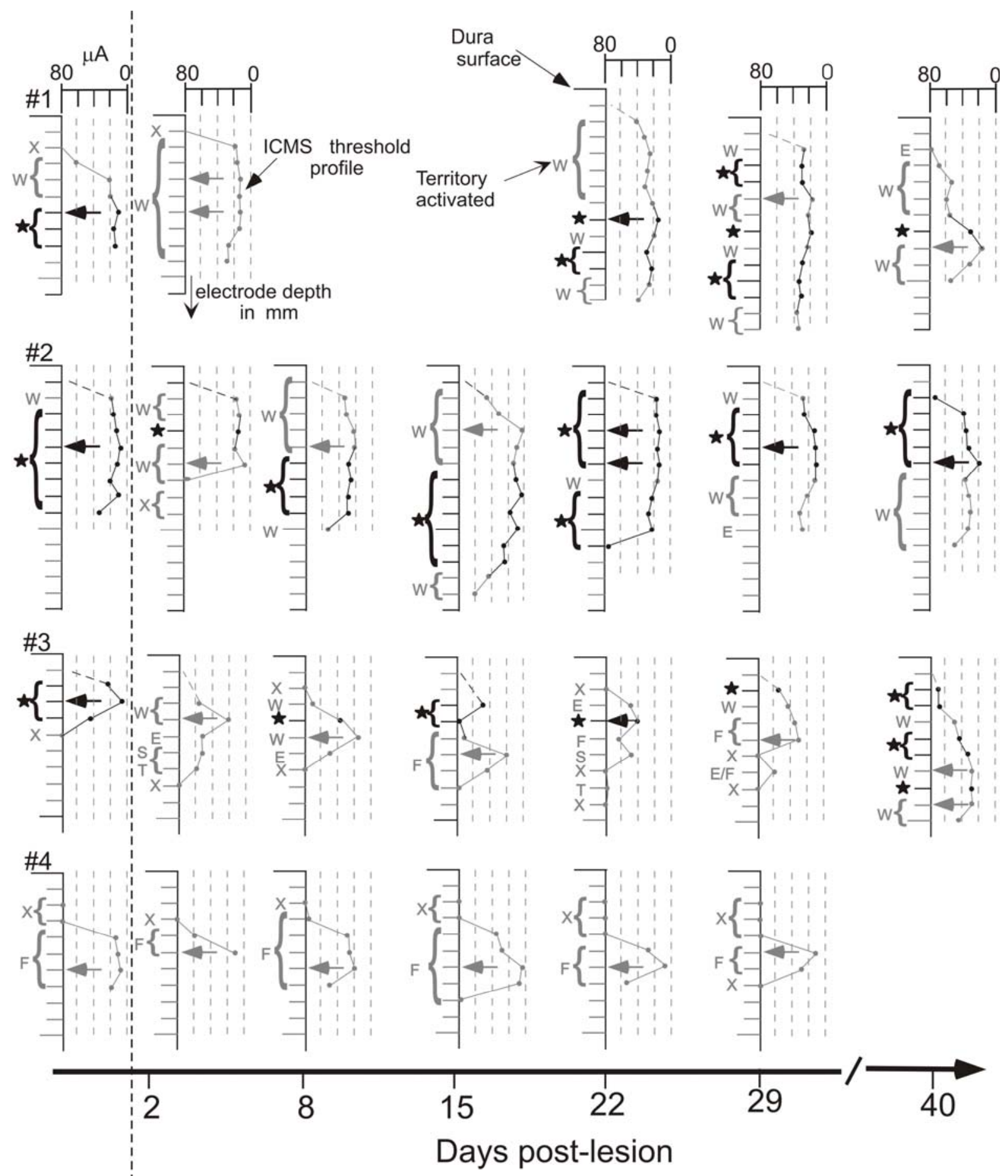

Fig. 3 (Schmidlin et al.) 


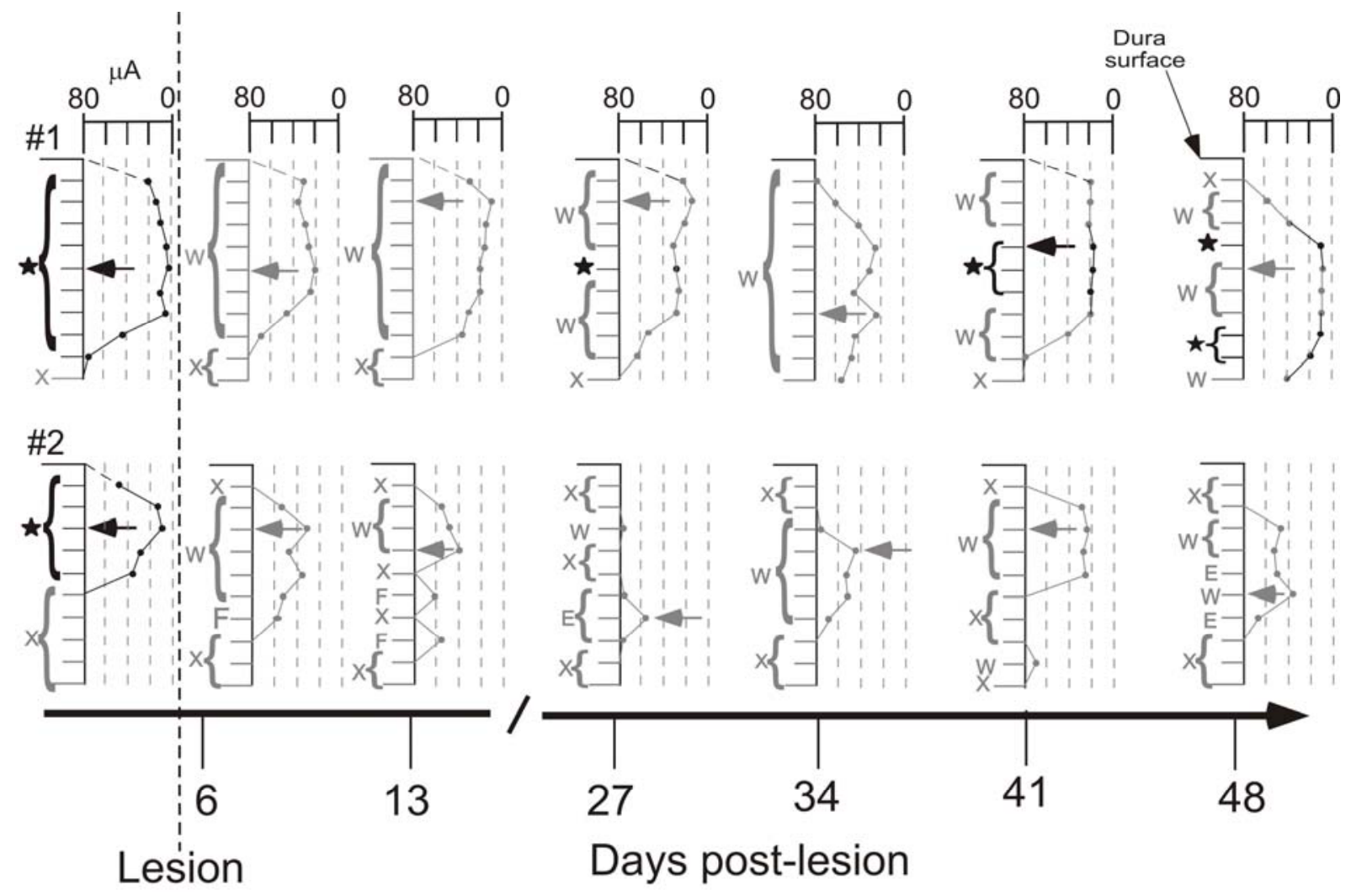

Fig. 4 (Schmidlin et al.) 